\title{
Growth and decay of large fluctuations far from equilibrium ${ }^{\dagger}$
}

\author{
SHRABANI SEN ${ }^{2}$, SYED SHAHED RIAZ ${ }^{1}$ and DEB SHANKAR RAY ${ }^{2, *}$ \\ ${ }^{1}$ Department of Chemistry, Belur Ramakrishna Mission Vidyamandira, Belur, Howrah 711202 \\ ${ }^{2}$ Department of Physical Chemistry, Indian Association for the Cultivation of Science, Jadavpur, \\ Kolkata 700032 \\ e-mail: pcdsr@iacs.res.in
}

\begin{abstract}
We have explored the weak noise limit of stochastic processes in nonlinear dissipative systems which admit of stable dynamical attractors in absence of noise. An interesting 'detailed balance' like condition in the steady state which is manifested in the time reversal symmetry between growth and decay of fluctuation far from equilibrium, similar to what is observed in thermally equilibrated systems, is demonstrated.
\end{abstract}

Keywords. Detailed balance; nonlinear dissipative systems; fluctuation-dissipation theorem.

\section{Introduction}

Equilibrium state is an unique macroscopic state of a thermodynamic system. This is characterised by the condition of detailed balance ${ }^{1}$ at a temperature determined by the thermal noise intensity and the damping constant related formally by the celebrated fluctuation-dissipation relation. ${ }^{2}$ A simple well known consequence of the detailed balance is the time-reversal symmetry ${ }^{3}$ between growth and decay of fluctuations (large of the order $>>\sqrt{D}, D$ being the diffusion coefficient) around equilibrium. Although large fluctuations of the dynamical variables are rare, they may, however, make their presence felt in a wide variety of phenomena involving chemical reactions, mutations in DNA, nucleation in phase transition, glycolytic oscillations, etc. The principle of detailed balance takes care of, on an average, a balance of input of energy into the system through thermal fluctuation and output of energy from the system due to dissipation in a thermodynamically closed system. Can such a detailed balance like condition be perceived in the steady state under far from equilibrium condition? While the equilibrium state is unique the steady states depending on the non-linearity of the system are, in general, multiple in number. The object of the present paper is to show that a nonlinear dissipative dynamical system characterized by a stable attractor, like limit cycle or strange attractor can exhibit such

${ }^{\dagger}$ Dedicated to the memory of the late Professor S K Rangarajan *For correspondence a condition in one of the steady states. An interesting offshoot of this condition is a mirror image symmetry between the growth of fluctuation of the dynamical variable away from the stable state in presence of noise and its return to the stable state along a relaxational path in absence of noise. We look for the conspicuous nature of these steady states and illustrate our analysis using auxiliary Hamiltonian technique in several model nonlinear dissipative dynamical systems having stable dynamical attractors in appropriate parameter space.

\section{Analysis of the steady state of a class of stochastic nonlinear dissipative systems}

To put the present analysis in an appropriate context we first consider the motion of a Brownian particle characterized by coordinate $x$ and momentum $y$ in a potential field $V(x)^{4}$

$$
\begin{aligned}
& \dot{x}=y \\
& \dot{y}=-V^{\prime}(x)-\gamma y[h(x, y)-1]+\eta(t) .
\end{aligned}
$$

Here $\gamma y[h(x, y)-1]$ is a nonlinear damping term, $\gamma$ being a constant. $h(x, y)$ is a function of the variables $x, y$ such that the system admits of a stable dynamical attractor in absence of noise term $\eta(t)$. For example, one may choose $h(x, y)=x^{2}+y^{2}$, which corresponds to Lienard system, a generalization of Van der pol oscillator ${ }^{10}$ when $V(x)$ is considered for a harmonic potential. is $\eta(t)$ a zero mean, Gaussian, delta correlated noise given by 


$$
\begin{aligned}
& \langle\eta(t)=0\rangle \\
& \left\langle\eta(t) \eta\left(t^{\prime}\right)\right\rangle=D \delta\left(t-t^{\prime}\right) .
\end{aligned}
$$

When $h(x, y)=0$, the dissipation is linear (for $\gamma<0$ ). $\eta(t)$ may be internal or external depending on whether the strength of noise $D$ is determined by the Einstein's relation between mobility and diffusion $D=\gamma k T$ or not, respectively. Here $\mathrm{k}$ is the Boltzmann constant and $T$ is the temperature.

The Fokker-Planck or Kramers equation corresponding to Langevin description (1) is given by

$$
\frac{\partial \rho}{\partial t}=L \rho+\gamma \frac{\partial}{\partial y}[(h(x, y)-1) y] \rho+D \frac{\partial^{2} \rho}{\partial y^{2}},
$$

where $\rho(x, y)$ is the probability density function in phase space. $L \rho$ corresponds to the Liouville dynamics

$$
V^{\prime}(x) \frac{\partial \rho}{\partial y}-y \frac{\partial \rho}{\partial x}
$$

Rewriting (5) in a form which takes care of both reversible liouville part and irreversible contribution as

$$
\frac{\partial \rho}{\partial t}=L \rho+\frac{\partial}{\partial y} J_{i r r}
$$

where

$$
-J_{i r r}=\gamma\{h(x, y)-1\} y \rho+D \frac{\partial \rho}{\partial y},
$$

The average heat exchange between the system and the reservoir can be calculated from the irreversible part $-J_{i r r}$ as

$$
\begin{aligned}
& \left\langle\frac{\mathrm{d} Q}{\mathrm{~d} t}\right\rangle=-\int_{-\infty}^{+\infty} \mathrm{d} y \int \mathrm{d} x \frac{\partial E}{\partial y} J_{i r r} . \\
& =\int \mathrm{d} x \int_{-\infty}^{+\infty} \mathrm{d} y \gamma y^{2}[h(x, y)-1] \rho+D \int \mathrm{d} x \int_{-\infty}^{+\infty} \mathrm{d} y y \frac{\partial \rho}{\partial y} .
\end{aligned}
$$

Here the integration ranges over the available phase space of the attractor. Carrying out integration by parts of the second term we obtain

$$
\left\langle\frac{\mathrm{d} Q}{\mathrm{~d} t}\right\rangle=-\gamma\left\langle y^{2}\right\rangle+\gamma\left\langle\varepsilon_{\mathrm{ex}}(x, y)\right\rangle-D
$$

where $\varepsilon_{\mathrm{ex}}=y^{2} h(x, y)$. The energy exchange between the system and the heat reservoir is thus contributed by three terms. First term denotes the average energy change due to linear term. The second one refers to the average energy change due to self-excitation of nonlinear dynamical origin and the third term corresponds to the average energy change due to thermal/ nonthermal noise. At the steady state $\langle\mathrm{d} Q / \mathrm{d} t\rangle=0$ and we have

$$
\gamma\left\langle y^{2}\right\rangle=\gamma\left\langle\varepsilon_{\text {ex }}(x, y)\right\rangle-D
$$

We now examine the following cases: (i) The noise is internal, i.e. one assumes the validity of the fluctuation-dissipation relation, $D=\gamma k T$ and the dynamical system is characterized by linear pumping, i.e. $h(x, y)=0$ and $\gamma>0$ in (2) (this is the reverse situation of linear damping). Equations (1) and (2) correspond to a linearly pumped stochastic system. One then arrives at an unphysical condition $-\gamma\left\langle y^{2}\right\rangle=k T$ from (11) and therefore the system can not reach equilibrium. (ii) On the other hand, when the system is a linearly damped stochastic system we have $h(x$, $y)=0$ and $\gamma<0$ in (11). One then recovers from (2) the standard equipartition result $1 / 2\left\langle y^{2}\right\rangle=1 / 2 k T$ at equilibrium. (iii) However, when the noise is internal and the system is nonlinearly damped, i.e. $h(x, y) \neq 0$ and $\gamma>0$ in (2) we obtain from (11) the following relation;

$$
\left\langle y^{2}\right\rangle=\left\langle\varepsilon_{\mathrm{ex}}(x, y)\right\rangle-k T .
$$

This situation is of special interest to us in this paper since this condition captures the special role of nonlinear dissipation in attaining the steady state of the dynamics. Absence of $\gamma$ indicates that the approach to the steady state is independent of its path of approach and the state is an unique state of the system. In this state the average energy loss due to linear dissipation is balanced by the average energy gain due to self excitation of the system and thermal noise due to bath in a unique way. The steady state expresses a detailed balance-like condition similar in spirit to what is expressed by fluctuation-dissipation relation. (iv) When the noise is external, the fluctuation-dissipation relation is not valid, i.e. $D \neq \gamma k T$, we reach a dynamical steady state given by the con- 
dition (11). This steady state is distinctly different from the previous one (12) in the sense that it depends on the path characterized by $\gamma$ along which the dynamical system moves to the steady state. Our object in this paper is to analyse the nature of the steady state implied by the condition (12).

\section{The weak noise limit of the stochastic process}

We begin with the note that the state of thermal equilibrium is characterised by the condition of detailed balance. A few years back Luchinsky and McClintok have demonstrated that there exists a time-reversal symmetry between growth and decay of fluctuation of dynamical variables at equilibrium which is a consequence of detailed balance. The large fluctuations can be studied in the weak noise limit of stochastic process using auxiliary Hamiltonian or path integral techniques. Luchinsky and McClintok ${ }^{5-7}$ have considered this weak noise limit of overdamped Brownian motion in a force field $K(x, t)$. The physical situation is governed by the following Fokker-Planck (FP) equation for probability density $\rho(x, t)$.

$$
\frac{\partial \rho(x, t)}{\partial t}=-\frac{\partial}{\partial x} K(x, t) \rho+\frac{D}{2} \frac{\partial^{2} \rho}{\partial x^{2}} .
$$

In the weak noise limit the diffusion coefficient $D$ is considered to be a smallness parameter such that $L t D \rightarrow 0, \rho(x, t)$ can be described by a WKB type approximation of the FP equation of the form $\rho(x, t)=Z(x, t) e^{W(x, t) / D} \cdot Z$ is a prefactor and $W(x, t)$ is the classical action satisfying a HamiltonianJacobi equation which can be solved by integration of the auxiliary Hamiltonian equation of motion

$$
\dot{x}=K+p, \dot{p}=\frac{\partial K}{\partial x} p .
$$

The auxiliary Hamiltonian is

$$
H_{\text {aux }}(x, p, t)=p K(x, t)+\frac{p^{2}}{2}
$$

with $p=\partial W / \partial x$ treated as a momentum of the auxiliary system. The origin of momentum is the inherent noise or fluctuation of the reservoir. In a thermally equilibrated system a large fluctuation of the variable $x$ implies a temporary departure from its stable state to some remote state (in presence of $p$ ) followed by a return to the stable state as a result of relaxation in absence of fluctuation. Luchinsky and McClintok have studied fluctuational and relaxational paths in analog electronic circuits and decay of fluctuations at equilibrium for $K(x)=-x^{3}+x$, when the system is driven away from stable fixed point $(-1$ or +1$)$. This symmetry, however, is not preserved when the unstable state of the system is chosen as the initial state or the system is acted upon by an additional external forcing. In both cases the system fails to be thermally equilibriated because of the loss of detailed balance.

We now turn to the central theme of the paper and enquire whether the presence of a stable dynamical attractor (limit cycle/strange attractor) in a dissipative nonlinear system can result in a symmetry between growth and decay of fluctuation far from equilibrium. Our motivation lies in exploring the possibility of realization of the unique steady state condition (12) as an analogue of equilibrium state. In what follows we employ Hamiltonian method ${ }^{5}$ to analyse the weak noise limit of the stochastic process (1) ${ }^{6,7}$ For this purpose we choose $h(x, y)=x^{2}+y^{2}$ and $V(x)=1 / 2 x^{2}$ for which the dynamical system admits of a stable limit cycle. In the weak noise limit $L t D \rightarrow 0$ the Fokker-Planck equation for probability density function $\rho(x, y, t)=z(x, y, t) \exp (W(x, y, t) / D)$ is now governed by the auxiliary Hamiltonian of the form $H_{\text {aux }}=-f p-g q+q^{2}$ where $p=\partial W / \partial x$ and $q=\partial W /$ $\partial y$ denote the auxiliary momenta signifying noise degree of freedom in the extended phase space. $f(x, y)$ and $g(x, y)$ are given by

$$
\begin{aligned}
& \dot{x}=y \equiv f(x, y) \\
& \dot{y}=\gamma\left\{1-\left(x^{2}+y^{2}\right)\right\} y-x \equiv g(x, y) .
\end{aligned}
$$

The auxiliary equations of motion are given by

$$
\begin{aligned}
& \dot{x}=y \\
& \dot{y}=\gamma\left\{1-\left(x^{2}+y^{2}\right)\right\} y-x-2 q \\
& \dot{p}=2 \gamma x y q+q \\
& \dot{q}=-p+\gamma\left\{\left(x^{2}+3 y^{2}\right)-1\right\} q .
\end{aligned}
$$


The fixed point $(0,0)$ of the dynamical system in absence of noise unstable and the system admits of $\eta(t)$ a stable limit cycle for $\gamma>$ (inset in figure 1). With the introduction of noise degree of freedom, the trajectory in the extended phase space moves away from the limit cycle to a state of large fluctuation (compared to strength of noise $p, q \sim 10^{-6}$ ) at $t=0$ as shown in figure 1 (dark line). The fluctuational path from the stable limit cycle to the state of large fluctuation can be considered as an optimal

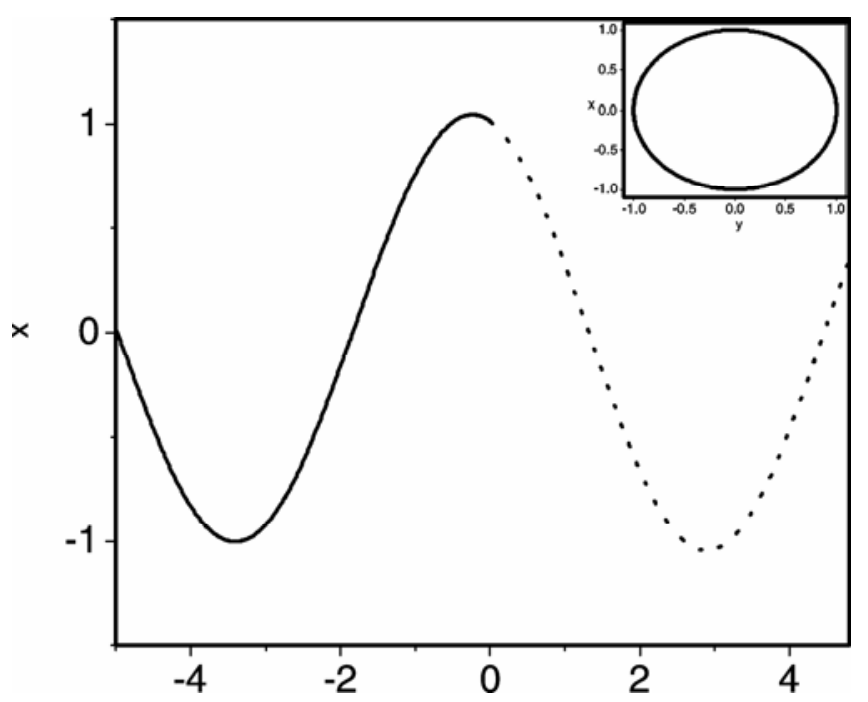

Figure 1. The mirror image symmetry between the path (solid line) for the fluctuation of $x$ as a function of time $t$ from a limit cycle (in the presence of noise) and the relaxational path (dotted line) towards the limit cycle in absence of noise. Inset (limit cycle in absence of noise) units arbitrary.

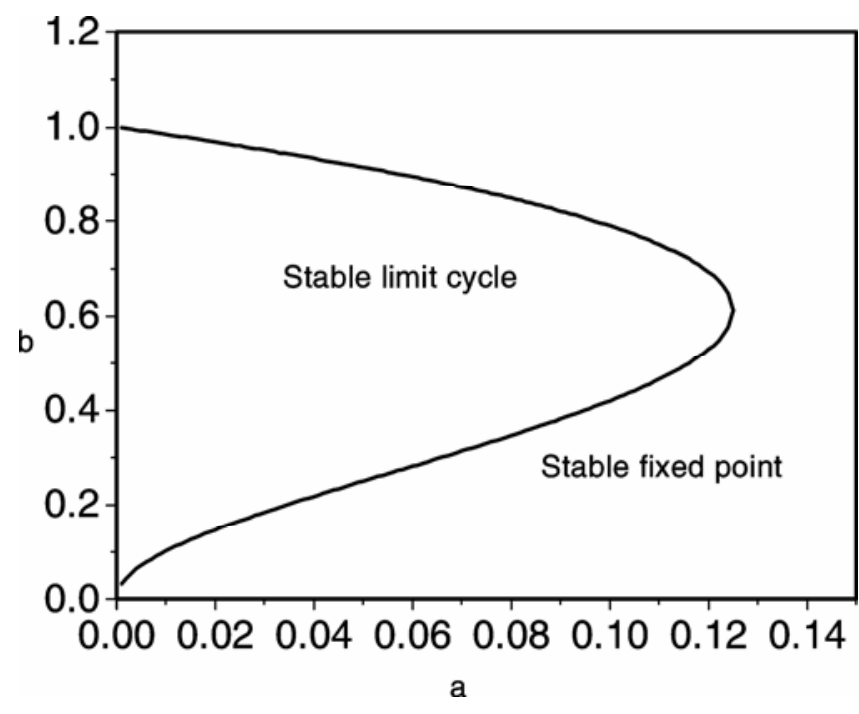

Figure 2. Stable limit cycle region and stable fixed point region for the Selkov model of glycolytic oscillator. path along which the system moves with overwhelming large probability. If the system is now allowed to relax in absence of noise variables $(p=q=0)$ to return to the limit cycle one observes a mirror image symmetry between growth and decay of fluctuation (dotted line in figure 1).

As a next example, we consider a prototype twovariable model described by $\dot{x}=f(x, y)+\eta(t)$; $\dot{y}=g(x, y)$ with

$$
\begin{aligned}
& f(x, y)=-x+a y+x^{2} y \\
& g(x, y)=b-a y-x^{2} y
\end{aligned}
$$

corresponding to Selkov model for glycolysis; ${ }^{8} x$ and $y$ being the concentrations of two chemical species, ADP and F6P, respectively. The equations of motion corresponding to the auxiliary Hamiltonian $H_{\text {aux }}=-f(x, y) p-g(x, y) q+p^{2}$ are given by

$$
\begin{aligned}
& \dot{x}=-x+a y+x^{2} y-2 p \\
& \dot{y}=b-a y-x^{2} y \\
& \dot{p}=p-2 x y p+2 x y q \\
& \dot{q}=-a p-x^{2} p+a q+x^{2} q .
\end{aligned}
$$

The linear stability analysis identifies stable limit cycle and stable fixed point regions in the parameter space $a, b>0$ as depicted in figure 2 . We choose the stable fixed point for $a=0.12$ and $b=0.2$ for the unperturbed system. In presence of noise degree of freedom the trajectory moves away from the stable fixed point to a remote state of large fluctuation at $t=0$ as shown in figure $3 \mathrm{a}$. When the system relaxes in absence of noise variables ( $p=q=0)$ to return to the stable state one observes a mirror image symmetry between the fluctuational and the relaxational path. This symmetry between the growth and the decay of fluctuations can also be found when the dynamical system is driven by the noise degree of freedom from the stable limit cycle for $a=0.08$ and $b=0.6$ followed by its relaxation. This is shown in figure $3 \mathrm{~b}$. The results in figures 1 and 3 are remarkably counterintuitive in view of the fact that these dynamical systems, in question, are under far from equilibrium condition. The origin of this time reversal symmetry under far from equilibrium condition, we believe, is the presence of stable limit cy- 
cle attractor due to the interplay of nonlinearity and dissipation such that the energy is injected in one region and extracted in another region of phase space. A limit cycle serves as a common boundary between them and implies a delicate balance between them ensuring strict periodicity and return to the stable manifold. In order to check this conclusion further we have studied another two-variable system, Lotka-Volterra predator-prey model with $f(x, y)=x-x y$ and $g(x, y)=x y-y$. This model allows stable oscillations but does not admit any limit
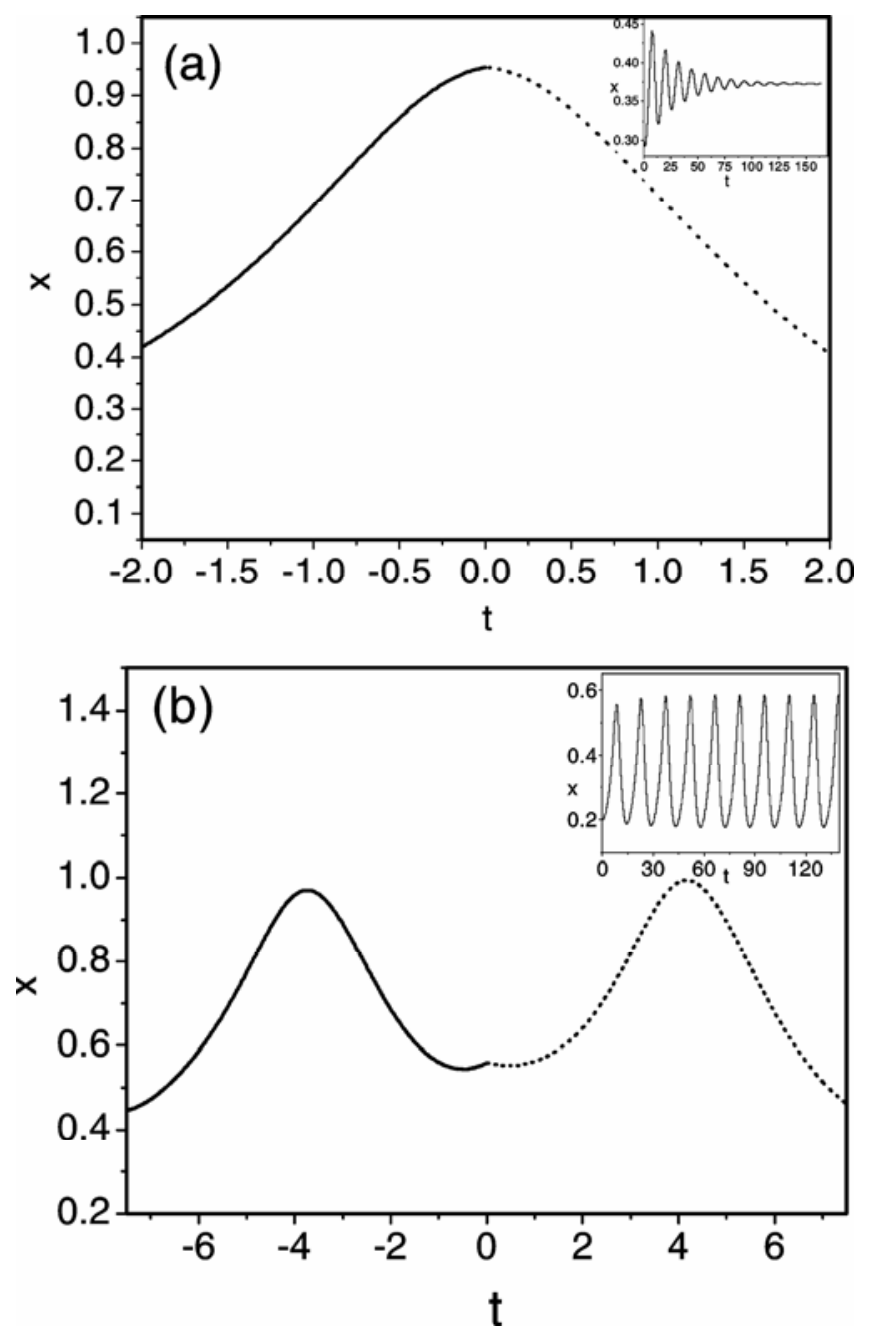

Figure 3. (a) The mirror image symmetry between the path (solid line) signifying the fluctuation of $x$ as a function of time $t$ from the steady state (in the presence of noise) and the relaxational path (dotted line) towards the steady state (in absence of noise) for the Selkov model of glycolytic oscillator. Inset (dynamics in absence of noise) units arbitrary. (b) Same as in figure 3a, but for the fluctuation from a limit cycle for the parameter set mentioned in the text. Inset sustained oscillation as a function of time in absence of noise. cycle. The weak noise limit of the corresponding stochastic dynamical system does not exhibit this symmetry between fluctuational and relaxational paths.

Can the above analysis be extended to chaotic attractors? To address this question we now consider the prototype three-variable Lorenz model, ${ }^{9}$ which has served as a traditional paradigm of nonlinear dynamics for decades. The stochastic equations are

$$
\begin{aligned}
& \dot{x}=\sigma(y-z)+\eta(t) \\
& \dot{y}=r x-y-x z \\
& \dot{z}=x y-b z,
\end{aligned}
$$

$\eta(t)$ being the external white noise as described earlier. The parameters $\sigma$ and $b$ are fixed at 10 and $8 / 3$, respectively while $r$ is varied. Depending on the values of $r$ several regions in phase space of the unperturbed system can be identified. ${ }^{10}$ The origin is globally stable for $r<1$. At $r=1$ the origin loses its stability by a supercritical pitchfork bifurcation and a symmetric pair of attracting fixed points is born. At a critical value of $r$,

$$
r_{H}=\sigma \frac{(\sigma+b+3)}{(\sigma-b-1)},
$$

fixed points lose stability through Hopf bifurcation. For the present purpose we have fixed the parameter space for the values $r=10$ (stable fixed point region), $r=28$ (strange attractor region) and $r=300$ (limit cycle region).

Proceeding exactly as in the earlier cases we derive the following auxiliary Hamiltonian equations of motion

$$
\begin{aligned}
& \dot{x}=\sigma(y-x)-2 u \\
& \dot{y}=r x-y-x z \\
& \dot{z}=x y-b z \\
& \dot{u}=\sigma u-r v+v z-y w \\
& \dot{v}=-\sigma u+v-x w \\
& \dot{w}=x v+b w .
\end{aligned}
$$



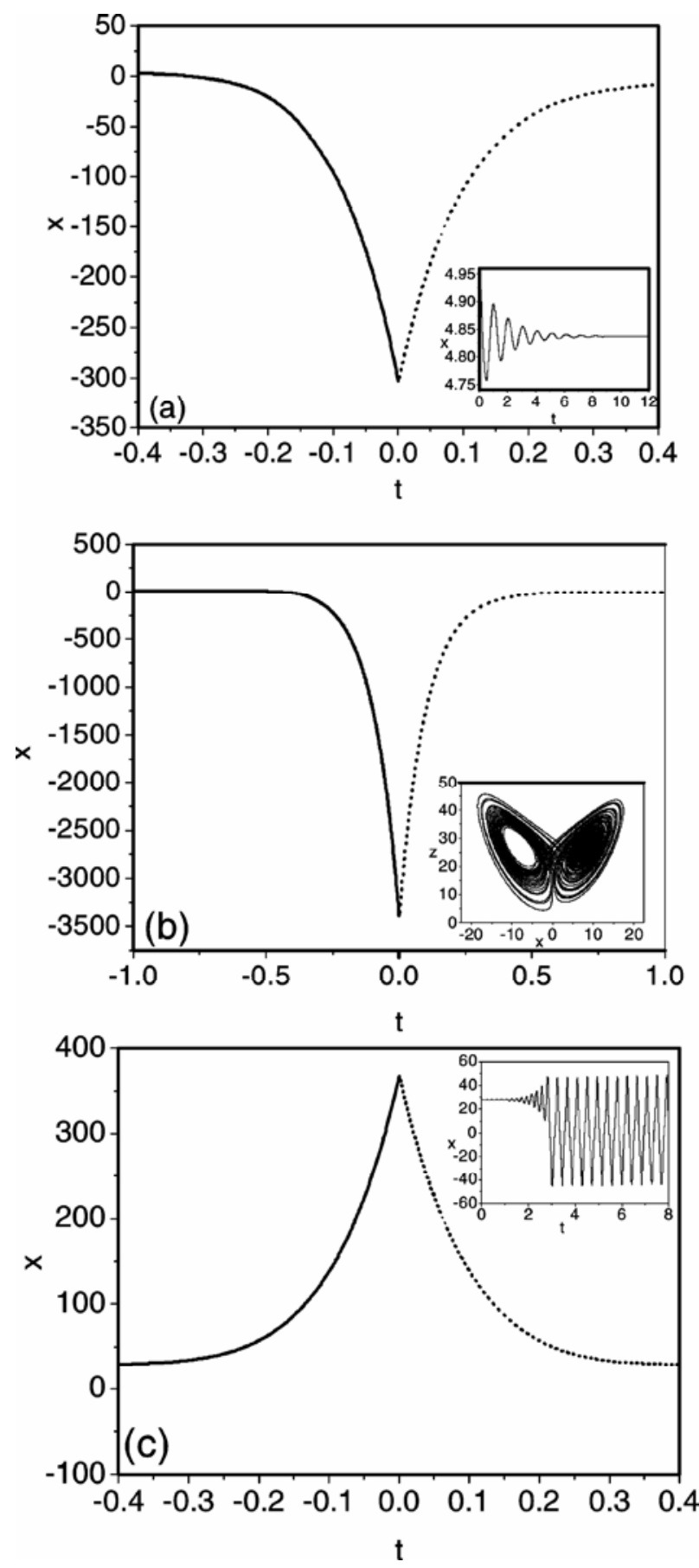

Figure 4. (a) The mirror image symmetry between the path (solid line) for the fluctuation of $x$ as a function of time $t$ from the steady state (in the presence of noise) and the relaxational path (dotted line) towards the steady state in absence of noise for the Lorenz model. Inset (approach to steady state in absence of noise) units arbitrary. (b) Same as in figure $4 \mathrm{a}$, but for fluctuation from a chaotic attractor for the parameter set mentioned in the text. Inset (strange attractor ( $z$ vs $x$ ) in absence of noise. (c) Same as in figure $4 a$, but for fluctuation from a limit cycle for the parameter set mentioned in the text. Inset (sustained limit cycle oscillation in absence of noise).
Here $u, v, w$ denote the auxiliary momenta signifying noise degree of freedom, conjugate to $x, y$ and $z$ respectively. In figure $4 a-c$ we display the numerical results on the growth and decay of large fluctuation for the three different phase space regions (stable fixed point, chaotic attractor and limit cycle regions) depicting the moving away of the trajectories along the fluctuational paths $(u, v, w \neq 0)$ and their return along the relaxational paths $(u, v, w=0)$. We find that the fluctuational and the relaxational paths obey the mirror image symmetry in the same way as before. The time reversal symmetry may be viewed as arising from a degeneracy between the projections of two different curves in an extended phase space onto the space of original variables. The degeneracy is not, however, robust against an applied force.

\section{Conclusion}

In conclusion, we have shown that an interplay of nonlinearity and dissipation in stochastic dynamical systems with stable attractors (like limit cycle, strange attractor) may give rise to an unique steady state. This state is conspicuous by the independence of its path of approach as a thermally equilibrated state and allows us to realize a detailed balance-like condition. This is reflected in the time reversal symmetry between growth and decay of fluctuation around the steady state similar to what is observed in thermally equilibrated systems and is a consequence of a delicate balance between energy gain due to self-excitation and energy loss due to dissipation in specified regions of phase space where the stable attractor (limit cycle or chaotic attractor) lies at the common boundary between them. In view of a very few results known for far-from-equilibrium condition (e.g. pattern formation, ${ }^{11}$ Jarzynski equality $^{12,13}$ and related fluctuation theorems), ${ }^{14}$ we believe that our observation on the time reversal symmetry, promises new relations involving correlation functions and transition rates and understanding the nature of non-equilibrium distribution functions and elucidating the nature of turbulence and biological systems.

\section{Acknowledgements}

Partial financial support from the Council of Scientific and Industrial Research (CSIR), Govt of India, is thankfully acknowledged. 


\section{References}

1. See, for example, Ma S K 1985 Statistical mechanics (Philadelphia, Singapore: World Scientific)

2. Callen H B and Welton T A 1951 Phys. Rev. 83, 34; Kubo R 1966 Rep. Prog. Phys. 29255

3. Luchinsky D G and McClintok P V E 1997 Nature (London) 389403

4. Kramers H A 1940 Physica 7 284; Hanggi P, Talkner P and Borkovec M 1990 Rev. Mod. Phys. 62 251

5. Freidlin M I and Wentzell A D 1984 Random perturbation in dynamical systems (NY: Springer)

6. Dykman M I and Krivoglaz M A 1984 Sov. Phys. Rev. 5 265; (ed.) I M Khalatmikov (NY: Harwood)
7. Graham R and Tel T 1984 Phys. Rev. Lett. 52 9; Kautz R L 1996 Rep. Prog. Phys. 59 935; Bag B C and Ray D S 2000 Phys. Rev. E62 4409; Bag B C and Ray D S 2000 Phys. Rev. 613223

8. Selkov E E 1968 Eur. J. Biochem 4 79; Kar S and Ray D S 2003 Phys. Rev. Lett. 90238103

9. Lorenz E N 1963 J. Atmos. Sci. 20 130; Haken H 1975 Phys Lett. A53 77; Ray D S 1984 Phys. Rev. A29 3440

10. Strogatz S H 1994 Nonlinear dynamics and chaos (New York: Addison Wesley)

11. Cross M C and Hohenberg P C 1993 Rev. Mod. Phys. 65851

12. Jarzynski C 1997 Phys. Rev. Lett. 782690

13. Liphardt J et al 2002 Science 2961832

14. Crooks G E 1999 Phys. Rev. E60 2721 